\title{
Minorities in a model for opinion formation
}

\author{
M. F. Laguna, Guillermo Abramson and Damián H. Zanette \\ Centro Atómico Bariloche, CONICET and Instituto Balseiro, \\ 8400 San Carlos de Bariloche, Río Negro, Argentina
}

(Dated: September 29, 2018)

\begin{abstract}
We study a model for social influence in which the agents' opinion is a continuous variable [G. Weisbuch et al., Complexity 7, 2, 55 (2002)]. The convergent opinion adjustment process takes place as a result of random binary encounters whenever the difference between agents' opinions is below a given threshold. The inhomogeneity in the dynamics gives rise to a complex steady state structure, which is also highly dependent on the threshold and on the convergence parameter of the model.
\end{abstract}

Keywords: Social systems, opinion formation

\section{INTRODUCTION}

The formation of public opinion is a complex phenomenon that affects almost all aspects of human life. In a population where each agent has an independent opinion, a typical situation when a common decision needs to be taken involves the exchange of information and points of view. As a result of these exchanges, people gradually change their opinions and a collective state emerges. In some cases, a single position is held by the population in the end. In others, a state of coexistence between diverging opinions may result and persist. In some regards, the emergence of a collective state is reminiscent of many other synchronization effects in populations with global coupling in the fields of physics, chemistry and biology [1, 2, 3]. The starting point of the present work is a model for opinion formation introduced by Weisbuch and coworkers [4]. The state of the agents is a continuous variable, representing an opinion, that evolves according to rules tending to homogenize the system. The conditions to reach consensus in this model were analyzed in previous works $[4,5]$, but several interesting properties were not studied. In particular, we analyze the transient state during which the formation of the collective opinion takes place, as well as the relevance of dynamical time scales in the formation of minorities. As an alternative to continuous opinions, discrete opinions have also been studied by Weisbuch and coworkers [4], and by ourselves in a previous work [6].

Let us consider a population of $N$ agents, each of them characterized by a continuous state variable, the "opinion," $x \in[0,1]$. Agents interact and update their opinions as a result of mutual influence in the following manner. At each time step $t$, two randomly chosen agents meet. If the difference between their opinions is smaller than a threshold, $\left|x(t)-x^{\prime}(t)\right|<u$, both change their opinions according to the rule:

$$
\begin{aligned}
x(t+1) & =x(t)+\mu\left[x^{\prime}(t)-x(t)\right], \\
x^{\prime}(t+1) & =x^{\prime}(t)+\mu\left[x(t)-x^{\prime}(t)\right],
\end{aligned}
$$

where $x$ and $x^{\prime}$ are the agents' opinions and $0<\mu<1$ is a convergence parameter [4]. That is, if the two opinions are already sufficiently close, they approach even more.
On the contrary, if the two opinions are not close enough, the agents keep their opinions unchanged. The convergence parameter $\mu$ controls the approach of the two differing opinions. The threshold $u$ is taken as constant in time and uniform across the whole population. Weisbuch and coworkers have shown that, for large threshold values $(u>0.3)$, consensus is achieved in the system, whereas for lower values of $u$ several opinions persist, the number of which follows a general functional dependence of the form $[1 / 2 u]$ on the threshold, where $[x]$ indicates the integer part of $x$. The role of $\mu$, they observe, is only that of controlling the speed of convergence towards the stationary state. Below, we explore a further role of this parameter.

The final product of the process is a set of clusters of opinion, each one of them consisting of a subpopulation of agents with identical opinions. This clusterization process is gradual and in principle achieved at infinitely long times, due to the continuous character of the opinion variable and the exponential approach consequence of Eqs. (11) and (2). Nevertheless, the formation of the final clusters is evident at finite times, because the distance between clusters is bounded from below by the threshold $u$. It is then possible to define a criterion for the formation of clusters as follows: two agents belong to the same cluster of opinions if the difference between them is less than certain finite value $u_{0}$, smaller than $u$. A criterion like this is also sensible from a realistic point of view, since it would be unreasonable to maintain that two opinions could differ in an arbitrarily small quantity. In the results shown in the present paper, we fix $u_{0}=0.001$. This value of $u_{0}$ limits the number of effective opinions to one thousand, which is large enough for the systems with $N=10^{3}$ to $5 \times 10^{5}$ used in our simulations.

The formation of the clusters, with the criterion given above, is complete at a time $t_{f}$ proportional to $N$. The simulation is stopped when no more activity is observed in the clusterization process. The final state consists of a number of "major" clusters and a number of "minorities," defined as clusters with less than $10 \%$ of the population. As will be discussed below, the major clusters are determined by the value of the threshold. These are the clusters analyzed by Weisbuch and coworkers [4]. The 


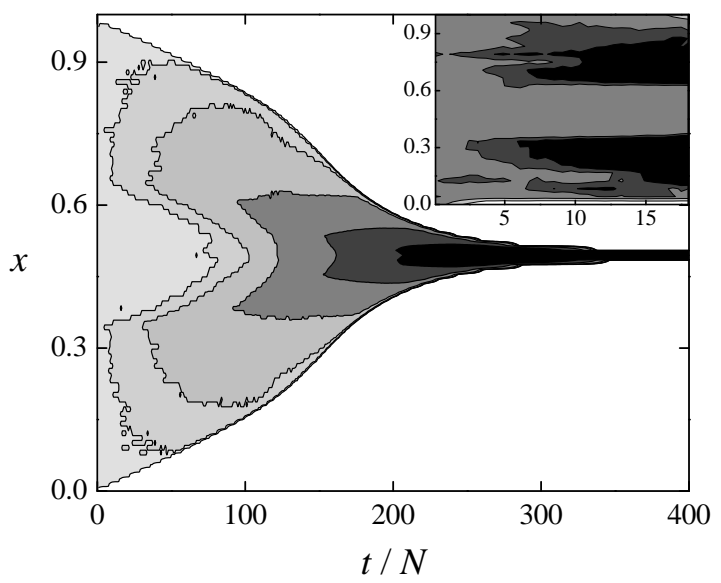

FIG. 1: Distribution of opinions as a function of time. The number of agents at each point of the graph is indicated in gray scale, with darker tones corresponding to higher densities. The inset shows in detail the first stage of the evolution, where the merging of the initial clusters can be seen. $N=5 \times 10^{5}, u=0.35, \mu=0.001$. The scale of grays is different in both plots, to facilitate the reading.

minorities - which, we stress, are not an artifact of the discretization criterion imposed on the opinion spaceare a byproduct of the dynamics, determined by the parameter $\mu$, through the dependence of the final state on the initial condition and on the transient steps.

\section{NUMERICAL RESULTS}

We analyze the dynamical behavior of the system starting from a random uniform distribution of opinions in the interval $[0,1]$. We begin with a value of the threshold large enough to induce the formation of a single major cluster. As time progresses, the region in opinion space occupied by the system shrinks, as shown in the time charts of Ref. 4]. Those plots, however, do not show the number of agents with the same value of opinion (the population of the clusters), which gives additional information about the clusterization process. To illustrate this feature of the evolution process, we plot in Fig. 1 the distribution of opinions as a function of time for a system with $N=5 \times 10^{5}, u=0.35$ and $\mu=0.001$. We have chosen this very small value of the convergence parameter to allow for access to the very initial steps of clusterization. In the figure, a density plot shows with darker colors the regions with more populated clusters.

In the inset of Fig. 1 we show the initial stage of the clusterization process. It can be seen that clusterization proceeds very fast to the formation of two main branches of opinion by the merging of smaller clusters, that appear as horizontal streaks in the inset. After this, the two main branches merge into the single final cluster, as shown in the main plot of Fig. [1]

The final state can be characterized by the number of clusters of opinion. For $u>0.3$ only one cluster is observed, whereas for lower values of $u$ several clusters persist, as studied in Ref. [4]. This situation takes place despite the fact that an opinion can propagate through the whole population. We have found that the final state depends, further, on the convergence speed through the parameter $\mu$. In Fig. 2 we plot the distribution of opinions as a function of the threshold for three typical values of $\mu$. We distinguish major clusters, with a population larger than $10 \%$ of the total (full dots), from minor clusters (empty dots). While the structure of the major clusters is the same in all the cases, the density of minor clusters near the extreme opinions 0 and 1 is higher for the larger values of $\mu$.

Numerical simulations performed over all the range of

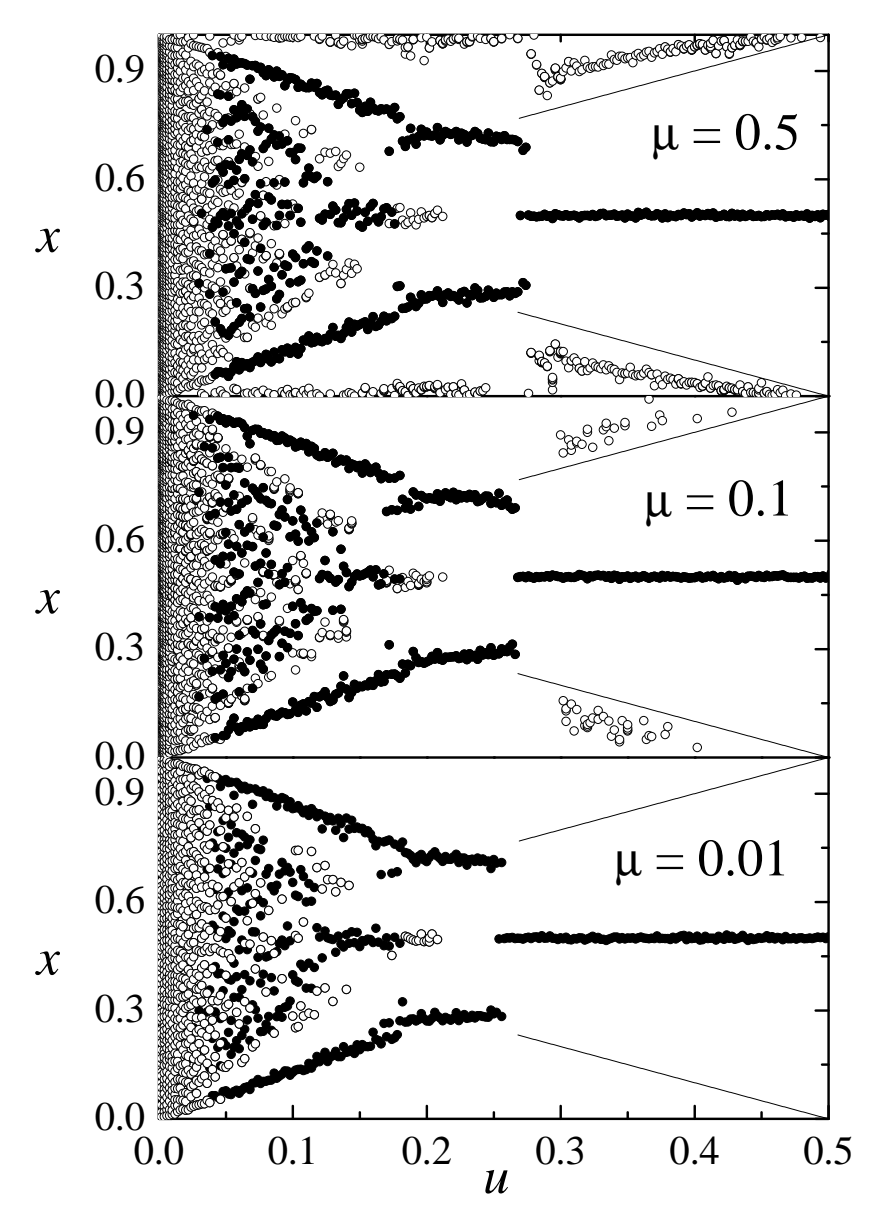

FIG. 2: Distribution of agent's opinions as a function of the threshold for three values of the convergence parameter, indicated in the figures. Empty dots indicate minor clusters, whereas full dots indicate major clusters, with population larger than 1000 agents, i.e. than $10 \%$ of the total population. The lines enclose the basin of attraction of the state with a single cluster. The results of a single realization are shown in each panel for each value of $u$. 


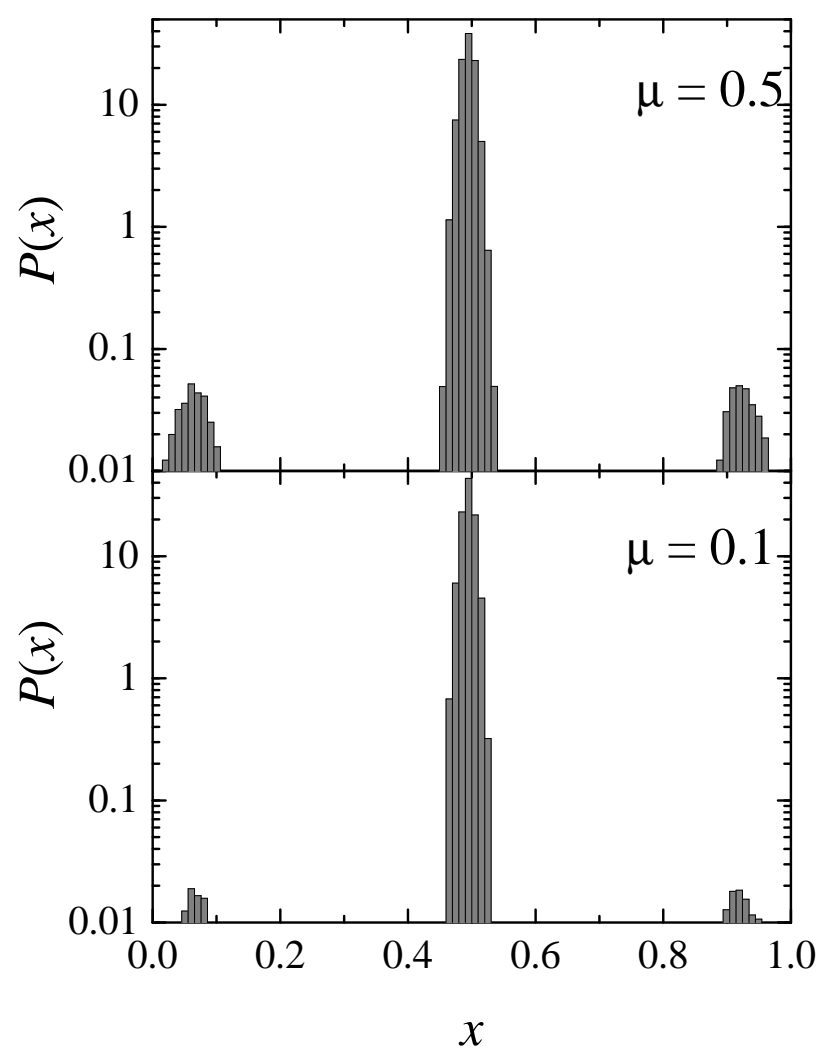

FIG. 3: Histogram of opinion for 2000 realizations with $N=$ 1000 and a fixed threshold, $u=0.35$. The central peak in both figures corresponds to major clusters, whereas the two lateral peaks are formed by minor clusters. In the upper panel the convergence parameter is big $(\mu=0.5)$ whereas in the lower panel $\mu=0.1$. Note the difference in the area of the minor clusters. Also observe that the distance between the clusters may be smaller than $u$, since the histograms are built from 2000 independent realizations.

thresholds show that the origin of the minor clusters is strongly dependent on the speed of the dynamics through the parameter $\mu$. For a given value of $u$, agents lying within a "basin of attraction" of radius $u$ from the budding clusters at an early stage of the evolution are almost certainly attracted to them, with a speed depending on $\mu$. In Fig. 2] we show the basin of attraction of the central opinion in the range of $u$ where a single cluster is obtained; the basins corresponding to states with more clusters are omitted to avoid clutter. Agents lying outside this basins cannot interact directly with the agents forming the clusters - since their opinions differ by more than $u$. However, agents with intermediate opinions may serve as bridges during the transient evolution, allowing agents from outside the basins to enter them. The availability of these intermediate agents strongly depends on $\mu$, since they ultimately tend to approach the clusters at a speed given by $\mu$. As a consequence the number of agents that remain frozen outside the basins increases with $\mu$. These agents, nevertheless, can interact with each other, and finally form a cluster by themselves, albeit a minor cluster, not a major one. These are the agents that appear outside the basins, in Fig. 22 for $\mu=0.1$ and 0.5 . The bottom panel of Fig. 2] corresponding to the slowest dynamics at $\mu=0.01$, does not exhibit these extreme opinions: the transient evolution has been slow enough to allow all of them to be incorporated into the central clusters.

The population of the minor clusters can be better appreciated in Fig. 3. where the results of 2000 independent realizations are averaged, for a fixed value of $u=0.35$ (one major cluster). When the convergence parameter is large (Fig. 3. top), the lateral clusters are, indeed, much bigger than the corresponding ones in the bottom plot, where the dynamics is slow and only a few agents remain outside the major cluster at the central opinion. It is evident, then, that the role of the parameter $\mu$ goes beyond than that of a speed of convergence parameter. It is able to modify the equilibrium state of the system.

The minorities located near the central opinion behave differently from those that, like the ones shown in Fig. [3] exist near the extremes. We can see in Fig. 2 that around $u=0.2$ there is a number of minorities that persist even for very low values of $\mu$ (refer to the empty dots near $x=0.5$ at $u \approx 0.2$ ). These clusters lie outside the basins of the two clusters that attract most of the population, so they are truly stationary. The intermediate opinions fail to move them to one of the major clusters during the early stages of the evolution because their central position makes them equally probable to move to either side in each individual interaction. Later, when the intermediate agents have disappeared, these minorities persist, and this happens for any value of the convergence parameter. The net effect of this in the phase portrait is that the transition from three clusters to two clusters results shifted towards larger values of $u$. The phenomenological rule, found in Ref. [4], that the number of clusters depends on $u$ as $[1 / 2 u]$, predicts a value of $u=1 / 6$ for this transition, lower than $u \approx 0.21$, as found in our results (Fig. 22). Certainly, this phenomenon occurs at lower thresholds also, affecting the other transitions as well and in the same direction. Its observation is, however, very difficult when the number of clusters is larger than three.

At lower values of $u$, where the stationary phase consists of more than two major clusters, it becomes manifest that the space of opinions is heterogeneous, i.e. the dynamics of an agent with a extremist opinion is different from the one with an intermediate opinion. In Fig. 4 we plot a histogram of opinions for 100 realizations and two values of the threshold, $u=0.17$ (three clusters), and $u=0.05$ (many clusters). In both cases, extremist clusters are overpopulated. Extreme clusters can be populated by the extremist agents that do not have access to the central clusters because of the small value 


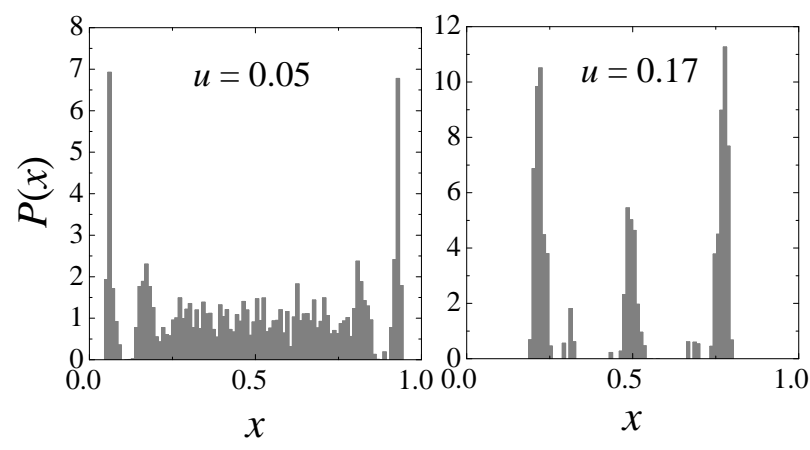

FIG. 4: Histograms of opinion for 100 realizations and two values of the threshold indicated in the figure, for $N=10^{4}$ and $\mu=0.1$. Note the larger population of the exterior clusters.

of the threshold. This gives advantage to the extreme clusters. This feature is not present in other models of opinion space, such as binary models [6], where there are no privileged opinions in this regard.

\section{CONCLUSION}

We have analyzed in detail a model of social influence and public opinion formation, previously presented by Weisbuch et al. 4]. The model treats opinion as a continuous scalar quantity, a simplification that can be of relevance in the process of decision making of very specific matters. The dynamics of the agents is a mechanism that approaches already nearby opinions, following Axelrod's 7] seminal ideas in the field of dissemination of culture. Our simulations confirm previous results for the model: large thresholds produce consensus in the population, while low thresholds do not, even when the randomness of the initial condition and the interaction mechanism allow, in principle, for the propagation of opinions throughout the whole population. Our new results provide details on the transient clusterization process, on the structure of the stationary phase controlled by the threshold parameter, and on the role of the convergence parameter on the final distribution of opinions. Even though we have not shown it, these results have proved to be very robust against noise of rather high amplitude.

The transient phase towards the clustered state allowed by the threshold shows progressive clusterization of the initial random state, by means of a collapse of the clusters that form in close regions of opinion. The speed of this process is certainly governed by the parameter $\mu$ which, besides, determines how many agents are left "frozen" outside the major clusters. If the dynamics proceeds slowly (small $\mu$ ), many agents whose opinions are initially far from what will become the consensus (farther than $u$, indeed), can nevertheless, by interacting with intermediate agents, be enabled to join the rest. If, on the other hand, the dynamics is fast, a large number of agents are left outside the consensus and end up forming extremist clusters of opinion.

As a result of this process, the final state of the system consists of a number of major clusters arranged, as a function of $u$, in the hierarchical structure shown in Fig. 2. A varying number of minorities, depending on the value of $\mu$, may also appear in the spaces between the major clusters in the final state. All clusters of opinion, major and minor, result more populated if they reside near one of the extremes of the opinion space. This is a consequence of the heterogeneity of opinion space in this kind of models, where the agents with extreme opinions are effectively less able to interact with others. For a given threshold $u$, agents with opinions in the middle range have in contrast more potential partners and tend to spread in the final state. Even if simplified, it is worthwhile to stress that this happens in many situations of collective decision making in human affairs, where extreme positions tend to remain irreconcilable with the majority.

In summary, despite its simplicity, the continuous opinion model with convergent dynamics displays a richness of features that can shed light over real system of opinion formation.

\section{ACKNOWLEDGMENTS}

M.F.L. thanks the Solid State Group of Centro Atómico Bariloche for the use of computational facilities.
[1] A. S. Mikhailov, Foundations of Synergetics I (Springer, Berlin,1990).

[2] Y. Kuramoto, Chemical Oscillations, Waves, and Turbulence (Springer, Berlin, 1984).

[3] A. T. Winfree, The Geometry of Biological Time (Springer, Berlin, 1980).

[4] G. Weisbuch, G. Deffuant, F. Amblard, J. P. Nadal, Com- plexity 7, No. 2, 55 (2002).

[5] M. Stone, Ann. of Math. Stat. 32, 1339 (1961).

[6] M. F. Laguna, G. Abramson and D. H. Zanette, Physica A (2003, in the press).

[7] R. Axelrod, The Complexity of Cooperation (Princeton University Press, 1997). 